\title{
LA DIVERSIDAD BIOLÓGICA EN LA ZONA RESERVADA ALLPAHUAYO - MISHANA, LORETO, PERÚ*
}

\author{
Juan José Rodríguez' ${ }^{1}$ Kalle Ruokolainen², Pekka Soini ${ }^{3}$ y Jukka Salo ${ }^{4}$
}

\begin{abstract}
RESUMEN
En esta colección de seis artículos de investigación se documenta la riqueza biológica de la Zona Reservada Allpahuayo - Mishana con información obtenida en cinco días de muestreo, durante el mes de noviembre de 2000, en el marco del curso "Uso de inventarios biológicos para revelar patrones de distribución geográfica de especies en la selva baja amazónica", organizado por el proyecto Diversidad Biológica de la Amazonía Peruana, Perú - Finlandia (BIODAMAZ), con el apoyo del Instituto de Investigaciones de la Amazonía Peruana (IIAP) y la Universidad Nacional de la Amazonía Peruana (UNAP). Treinta y tres investigadores, divididos en seis grupos de investigación, reportan 49 especies de Melastomatáceas, 41 especies de palmeras, 71 especies de helechos, 152 especies de aves, 49 especies de anfibios, 17 especies de reptiles, 15 familias de Coleópteros y 22 familias de Himenópteros. Se estudian las relaciones de la distribución y abundancia de las especies y los hábitats que ocupan: bosque de varillal (seco, semi seco y húmedo), bosque de chamizal, formaciones geológicas Pebas, bosque de terraza, bosque de colina, y quebradas. Los índices de Sørensen y Steinhaus fueron aplicados para expresar diferencias y semejanzas entre la composición de especies. Estos patrones de diferencias y semejanzas fueron visualizados a través de ordenaciones con el método de coordenadas principales. Las correlaciones de estos patrones con variación de hábitat fueron probadas con la prueba de Mantel.
\end{abstract}

Palabras clave: Abundancia, Amazonía peruana, distribución geográfica de especies, hábitat, inventarios biológicos, patrones de diferencias y semejanzas, Zona Reservada Allpahuayo - Mishana.

\begin{abstract}
This collection comprised of six research papers documents the biological richness of the Allpahuayo-Mishana Reserved Zone, with information obtained during five days of sampling during November 2000, as part of the "Use of biological inventories to reveal geographic distribution patterns of lowland Amazonian species" course organized by the Peru-Finland Biological Diversity of the Peruvian Amazon project, (BIODAMAZ), with the support of the Peruvian Amazonian Research Institute (IIAP) and the Peruvian Amazonian National University (UNAP). Thirty-three researchers divided into six research groups reported 49 species of Melastomataceae, 41 species of palms, 71 species of ferns, 152 species of birds, 49 species of amphibians, 17 species of reptiles, 15 families of Coleoptera and 22 families of Hymenoptera. Relationships between the distribution and abundance of the species and their habitats are studied, including forest habitats such as white-sand forests (dry, semi dry, humid and low), forests on Pebas geological formation, terrace forests, forest on hilly areas and brook gallery forests. The Sørensen and Steinhaus indices were used to express the similarities and differences between the
\end{abstract}

* Referencia bibliográfica del artículo: Rodríguez, J.J., Ruokolainen, K. \& Soini, P. 2002. La diversidad biológica de la Zona Reservada Allpahuayo - Mishana, Loreto, Perú. En: Rodríguez, J.J., Ruokolainen, K. \& Soini, P. (eds.). La diversidad biológica de la Zona Reservada Allpahuayo - Mishana, Loreto, Perú: Relaciones entre distribución, abundancia y hábitats. Folia Amazónica 14 (1-2): NÚMEROS DE PÁGINA.

1 Proyecto Diversidad Biológica de la Amazonía Peruana - BIODAMAZ, Perú - Finlandia. Av. Abelardo Quiñones km 2.5, Iquitos, Perú. Correo electrónico: biodamaz@iiap.org.pe

2 Proyecto Diversidad Biológica de la Amazonía Peruana - BIODAMAZ, Perú - Finlandia. Av. Abelardo Quiñones km 2.5, Iquitos, Perú / Departamento de Biología, Universidad de Turku, 20014 Turku, Finlandia.

3 Proyecto Diversidad Biológica de la Amazonía Peruana - BIODAMAZ, Perú - Finlandia. Av. Abelardo Quiñones km 2.5, Iquitos, Perú / Programa de Investigación para el Aprovechamiento Sostenible de la Biodiversidad - PBIO, Instituto de Investigaciones de la Amazonía Peruana - IIAP. Av. Abelardo Quiñones km 2.5, Iquitos, Perú.

4 Proyecto Diversidad Biológica de la Amazonía Peruana - BIODAMAZ, Perú - Finlandia. Av. Abelardo Quiñones km 2.5, Iquitos, Perú / Departamento de Biología, Universidad de Turku, 20014 Turku, Finlandia. 
species composition. These patterns of similarities and differences were visualized through ordination according to the principal coordinates method. The correlations of these patterns with habitat variation were tested with the Mantel test.

Key words: Abundance, Allpahuayo-Mishana Reserved Zone, biological inventories, geographic distribution of species, habitat, patterns of differences and similarities, Peruvian Amazon.

\section{INTRODUCCIÓN}

Durante los días 13 a 25 de noviembre de 2000, el proyecto Diversidad Biológica de la Amazonía Peruana, Perú - Finlandia (BIODAMAZ) ${ }^{5}$, con el apoyo del Instituto de Investigaciones de la Amazonía Peruana (IIAP) y la Universidad Nacional de la Amazonía Peruana (UNAP), organizó el curso "Uso de inventarios biológicos para revelar patrones de distribución geográfica de especies en la selva baja amazónica", orientado a estudiantes de pre y post grado en ciencias biológicas y afines. El curso tuvo una parte teórica y una parte práctica en la Zona Reservada Allpahuayo - Mishana (ZRAM), Loreto, en los alrededores de la Estación Biológica Allpahuayo. El objetivo de este evento fue capacitar a estudiantes en métodos de inventarios biológicos, utilizando grupos indicadores como una herramienta para establecer patrones de distribución basados en conocimientos biogeográficos y geoecológicos de la selva baja amazónica.

\section{2. ÁREA DE ESTUDIO}

La ZRAM está ubicada en la "Ecorregión Napo", considerada una de las ecoregiones globalmente sobresalientes debido a que posee la mayor diversidad de especies, para muchos taxa a nivel mundial, y está clasificada como de alta prioridad a escala regional para su conservación. Se caracteriza por presentar gran riqueza de ecosistemas, incluyendo formaciones vegetales sobre arena blanca, como "varillales y "chamizales", que podrían explicar, junto con posibles efectos de aislamiento geológico causado por diferentes épocas de erosión, sedimentación y separación de áreas fluviales, la diversidad biológica de la zona, contando con numerosas especies únicas, raras o de distribución restringida.

El relieve general de la ZRAM corresponde a la categoría de tierras bajas aluviales, más o menos disectadas, con terrenos no inundables y algunas zonas de colinas bajas (100-130 msnm), planicies de arenas blancas, con buen y mal drenaje, pequeñas áreas de pantanos y también terrenos estacionalmente inundables en las márgenes del río Nanay. Predominan las formaciones de arenas blancas cuarzíticas en terrazas medias y altas. Estas tierras son probablemente de origen muy antiguo y han sido recicladas muchas veces (están muy meteorizadas) (Räsänen et. al., 1998).

Los suelos de estas tierras se caracterizan por sus limitaciones muy severas o extremas, que los hacen inapropiados para propósitos agropecuarios o forestales; son muy pobres en nutrientes, muy ácidos y en ocasiones con mal drenaje, ocasionado por el afloramiento del "horizonte espódico", una capa impermeable de arena compactada con materia orgánica y óxidos de hierro. Suelos con mal drenaje también se encuentran en las terrazas bajas de la margen derecha del Nanay, y en los márgenes y cabeceras de algunas quebradas. Parte de estos suelos son estacionalmente inundables por las aguas del río Nanay. Estos terrenos son de origen aluvial más reciente.

Existe, finalmente, una porción de tierras planas o ligeramente onduladas, y colinas arcillosas, conformadas por sedimentos aluviales muy antiguos, aunque actualmente no están sujetos a inundaciones. La mayoría de estas tierras se caracterizan por presentar buena profundidad efectiva, textura franca a franco arcillosa, de reacción fuertemente ácida ( $\mathrm{pH}$ 5.0-5.3), con proporciones de aluminio intercambiable que varían desde niveles bajos a

5 Proyecto Diversidad Biológica de la Amazonía Peruana (BIODAMAZ) es un convenio entre los gobiernos del Perú y de Finlandia ejecutado conjuntamente por el Instituto de Investigaciones de la Amazonía Peruana (IIAP) y por el consorcio finlandés formado por la empresa de consultorías ambientales, Biota BD Oy, y por la Universidad de Turku. 
altos. Las dotaciones de materia orgánica, nitrógeno y potasio varían de baja a media, y los niveles de fósforo disponible son bajos. En algunas de estas zonas afloran en la superficie las arcillas de la formación geológica conocida como "Formación Pebas", con buen contenido de nutrientes debido a la presencia de fósiles de moluscos marinos (IIAP, 1996).

La zona climática de la ZRAM es de selva tropical lluviosa, caracterizada por precipitaciones anuales altas, entre 2500 y $3000 \mathrm{~mm}$, y temperaturas medias anuales altas, mayores de $26{ }^{\circ} \mathrm{C}$. Los cambios climáticos estacionales son poco apreciables (no existe estacionalidad marcada), y bastante variables, dependiendo más de la precipitación pluvial que de la temperatura. La humedad atmosférica es casi constante, variando de $80 \%$ hasta $100 \%$. Ocasionalmente, durante la estación más seca, de junio y julio, ocurren períodos de descenso moderado de las temperaturas (que bajan hasta 14 ó $15^{\circ} \mathrm{C}$ ), provocados por los vientos del hemisferio sur (Marengo, 1998).

\section{ESTUDIOS REALIZADOS}

Para los presentes trabajos, se realizaron investigaciones en tres familias de plantas: Melastomataceae, Arecaceae (palmeras) y Pteridophyta (helechos); y tres grupos de animales: aves, anfibios e insectos. Los investigadores hicieron inventarios de estos grupos y buscaron relaciones entre su distribución, abundancia y los diferentes tipos de hábitat en que se encuentran. Para ello, fijaron parcelas, transectos, estaciones, puntos de conteo, observaciones (en el caso de aves), y tomaron la información necesaria para procesar los datos.

Este informe compila los trabajos realizados en la ZRAM y documenta la gran riqueza de especies biológicas registradas en tan solo cinco días de muestreo.

\section{AGRADECIMIENTOS}

Nuestro agradecimiento al proyecto BIODAMAZ, Perú - Finlandia, por la oportunidad de realizar estos estudios.

\section{BIBLIOGRAFÍA}

IIAP. 1996. Zonificación Ecológica Económica del Área de Influencia de la Carretera Iquitos - Nauta. Documento Técnico. CIGAP-IIAP. 26 pp.

MARENGO, J.A. 1998. Climatología de la zona de Iquitos, Perú. En: Kalliola, R.; Flores, Paitán, S. (eds.). Geoecología y desarrollo amazónico: estudio integrado en la zona de Iquitos, Perú. Annales Universitatis Turkuensis Ser. A II 114: 37-57.

RÄSÄNEN, M.; LINNA, A.; IRION, G.; REBATA, L.; VARGAS R.; WESSELINGH, F. 1998. Geología y geoformas de la zona de Iquitos. En: Kalliola, R.; Flores, Paitán, S. (eds.). Geoecología y desarrollo amazónico: estudio integrado en la zona de Iquitos, Perú. Annales Universitatis Turkuensis Ser. A II 114: 59-137. 